\title{
Behavioral Approach to Robustness Analysis
}

\author{
Fernando Paganini Raffaello D'Andrea John Doyle \\ Electrical Engineering, M/S 116-81 \\ California Institute of Technology \\ Pasadena, CA 91125
}

\begin{abstract}
This paper introduces a general and powerful framework for modeling and analysis of uncertain systems. One immediate concrete result of this work is a practical method for computing robust performance in the presence of norm-bounded perturbations and both norm-bounded and white-noise disturbances.
\end{abstract}

\section{Introduction}

This paper builds on, extends, and unifies several related methodologies, including behavioral descriptions of systems [17], Integral Quadratic Constraint (IQC) [8], linear fractional transformation (LFT), and $\mu[11]$ frameworks for uncertainty descriptions, set characterizations of white noise [12], and linear matrix inequality (LMI) computation.

The LFT and $\mu$ frameworks have allowed for descriptions of uncertainty including norm-bounded perturbations and uncertainties not captured by IQCs, such as real parameter variations. The IQC formulation, on the other hand, allows for a more general class of quadratic constraints, such as those appearing in set characterizations of white noise. It is shown here how the behavioral extension to the LFT setup provides a complete mapping for these general IQCs, therefore allowing a general analysis framework with all the desirable features. Finally, reducing our solution to LMIs yields an attractive computational scheme that corresponds to what is available for standard robust performance problems.

In particular, this framework provides robust performance tests in the presence of norm bounded perturbations and white noise disturbances, which has been referred to as the Robust $\mathcal{H}_{2}$ performance problem. Most activity in the area of robust control with $\mathcal{H}_{2}$ performance specifications has fallen in the category of the socalled mixed $\mathcal{H}_{2} / \mathcal{H}_{\infty}$ problem (see [2] [18], [6], and [16]) which is equivalent to ensuring nominal $\mathcal{H}_{2}$ performance with robust stability under norm bounded perturbations. Some recent work ([15], [5]) has addressed the robust performance issue, by considering the worst-case response of the uncertain system to an impulse. The main contribution of our framework is that by incorporating approximate set characterizations of white noise which fit the LFT framework, the worst-case response under white noise is directly assessed, and is shown to be no harder than standard $\mu$ techniques for assessing robust $\mathcal{H}_{\infty}$ performance; moreover, the machinery is general enough to deal with mixed pexformance specifications.

\section{Background}

\subsection{Uncertainty and LFTs}

The major theme of robust control has been to explicitly consider descriptions of uncertainty in the analysis and design of control systems. There are various sources of uncertainty, such as parametric uncertainty, unmodeled dynamics, unmodeled nonlinearities and disturbances. A convenient approach to unify the descriptions of uncertainty together with other elements of control theory in a simple common mathematical language is the linear fractional transformation paradigm. LFTs are a class of general feedback loops, and are depicted in Figure 1. In principle, $G$ and $\Delta$ are arbitrary maps, and the closed loop map from $u$ to $y$ is given by

$$
\Delta * G \triangleq G_{22}+G_{21} \Delta\left(I-G_{11} \Delta\right)^{-1} G_{12}
$$

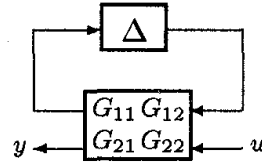

Figure 1: Linear Fractional Transformation

whenever the inverse is well defined.

When describing uncertainty in this framework, $\Delta$ will be denoted by $\Delta_{u}$ and will represent a structured uncertainty operator

$$
\Delta_{u}=\operatorname{diag}\left[\delta_{1} I_{r_{1}}, \ldots, \delta_{L} I_{r_{L}}, \Delta_{L+1}, \ldots, \Delta_{L+F}\right]
$$

The blocks in $\Delta_{u}$ can be used to describe real parameters, linear time invariant dynamical blocks, and more generally linear time varying or nonlinear perturbations. In each case, there is a restricted class $\Delta_{\mathrm{u}}$ of allowed perturbations. For a tutorial description of this, the reader is referred to [3].

In this paper we will be working in discrete time, and restrict ourselves to linear uncertainty blocks. The results could be extended to include nonlinear operators in the full blocks. We will denote by $l_{2}^{m}$ the space of vector $\left(\mathbb{R}^{m}\right.$ or $\mathbb{C}^{m}$-valued) square summable sequences, $l_{2}=l_{2}^{1}$, and by $\mathcal{L}\left(l_{2}^{m}\right)$ the set of linear, bounded operators in $l_{2}^{m}$. So the most general class of perturbations we will consider is $\delta_{i} \in \mathcal{L}\left(l_{2}\right), \Delta_{L+j} \in \mathcal{L}\left(l_{2}^{m_{j}}\right), 1 \leq i \leq L, 1 \leq j \leq F$.

The $G$ matrix is typically a finite dimensional LTI system $G(\lambda)$, where $\lambda$ is the delay operator. We can also exploit the fact that a state space realization of $G$ is in fact an LFT on the delay operator, and obtain an LFT representation of the uncertain system of the form $\Delta_{S} * M$, where $M=\left[\begin{array}{cc}A & B \\ C & D\end{array}\right]$ is a constant matrix. The augmented structure $\Delta_{S}=\operatorname{diag}\left[\lambda I \Delta_{u}\right]$ is still of the form described by (2), where now $\delta_{1}$ is taken to be $\lambda$. We shall consider both types of descriptions in what follows, and use the generic notation $\Delta$ whenever the distinction is irrelevant.

\subsection{The Behavioral Approach}

The behavioral setting for dynamical systems can be characterized by the fact that all variables are considered a priori on an equal footing, without a distinction between inputs and outputs, and the behavior is defined as a subset of the possible time trajectories. Behavioral descriptions arise naturally when modeling physical systems from first principles, where physical laws such as mass and energy balances are more naturally thought of as relations between variables than as input/output maps. Interconnections of subsystems are reduced to intersecting constraints. We will find this point of view of implicit constraints very suitable to pose general robustness analysis questions.

Of the many characterizations of behavioral systems, in Figure 2 we single out the output nulling $(\mathrm{ON})$ representation:

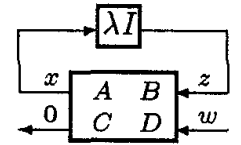

Figure 2: $\mathrm{ON}$ representation

Here the vector $w$ contains all the manifest variables. In the case of regular representations ( $D$ is full row rank), a valid input/output 
partition is characterized as follows: if after permutation of the order of variables we can partition $D=\left[D_{1} D_{2}\right]$, with $D_{1}$ square and invertible, the first group of variables is a valid output choice.

The reader is referred to [17] and [3] for detailed expositions of the ideas outlined above.

\subsection{Uncertain Behavioral Descriptions}

In [4], the robust control and behavioral paradigms are unified by incorporating structured uncertainty into the description of behavioral systems. The idea is to extend the $\mathrm{ON}$ representation and define uncertain behaviors as kernels of operators obtained by an LFT between a matrix and an uncertainty structure. Once again we can consider two versions, the kernel of $\Delta * G$ where $G$ is a dynamical system, or the kernel of $\Delta_{S} * M$, where $M$ is a constant matrix. The second case directly is shown in Figure 4, and is called a Generalized Output Nulling (GON) representation of the uncertain behavior. The issues of regularity and valid $\mathrm{I} / \mathrm{O}$ partitions can be extended directly, and are discussed in [4].

\section{The Framework for Robustness Analysis}

In the following discussion we shall make use of the following facts; the proof is omitted (refer to [13]).

Lemma 1 Let $z, v \in l_{2}^{d}$. The following are equivalent:

$$
\begin{aligned}
& \text { (i) }\|v\|_{2}^{2}-\|z\|_{2}^{2} \geq 0 \\
& \text { (ii) } \exists \Delta \in \mathcal{L}\left(l_{2}^{d}\right),\|\Delta\| \leq 1, \Delta v=z
\end{aligned}
$$

Lemma 2 Let $z, v \in l_{2}^{d}$. The following are equivalent:

$$
\begin{aligned}
& \text { (i) } \sum_{t=-\infty}^{\infty} v(t) v(t)^{*}-z(t) z(t)^{*} \geq 0 \\
& \text { (ii) } \forall \eta \in \mathrm{C}^{d},\left\|\eta^{*} v\right\|_{2} \geq\left\|\eta^{*} z\right\|_{2} \\
& \text { (iii) } \exists \delta \in \mathcal{L}\left(l_{2}\right),\|\delta\| \leq 1, \delta I_{d} v=z
\end{aligned}
$$

\subsection{Robust stability and robust performance}

We begin by reviewing the issue of robust stability of a standard LFT interconnection as shown in Figure 3(a), when $\Delta_{u}$ is allowed to vary in a normalized ball of structured uncertainty,

$$
\mathbf{B}_{\boldsymbol{\Delta}_{\mathbf{u}}} \triangleq\left\{\Delta_{u} \in \boldsymbol{\Delta}_{\mathbf{u}},\left\|\Delta_{u}\right\| \leq 1\right\}
$$

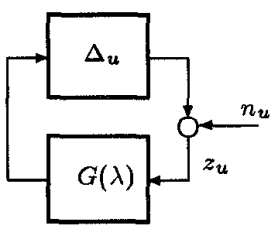

(a)

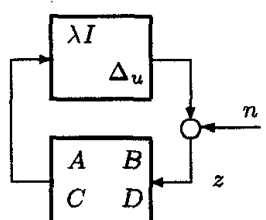

(b)
Figure 3:

Assuming the nominal system $G(\lambda)$ is stable, robust stability means informally that for any $\Delta_{u} \in \mathbf{B}_{\boldsymbol{\Delta}_{u}}$ the loop cannot sustain signals with arbitrarily small amounts of injected noise $n_{u}$. A (slightly stronger) definition follows.

Definition 1 The feedback interconnection of Figure $s(a)$ has uniform robust stability if $G(\lambda)$ is stable, and there exists $\epsilon>0$ such that if $z_{u} \in l_{2},\left\|z_{u}\right\|=1, \Delta_{u} \in \mathbf{B}_{\mathbf{\Delta}_{u}}$, then $\left\|\left(I-\Delta_{u} G\right)\left(z_{u}\right)\right\| \geq \epsilon$.

When dealing with state space LFTs as in Figure 3(b) the previous definition can be adapted to encompass both the nominal stability and the robustness, by substituting $G$ by $M, z_{u}$ by $z$, and $\mathbf{B}_{\Delta_{\mathbf{u}}}$ by $\mathbf{B}_{\mathbf{\Delta}_{\mathbf{s}}}=\left\{\operatorname{diag}\left[k \lambda \Delta_{u}\right],|k| \leq 1,\left\|\Delta_{u}\right\| \leq 1\right\}$

In fact, it can be shown (see Theorem 3 ) that this definition then implies the nominal (internal) stability condition $\rho(A)<1$ and the uniform robust stability of the corresponding $\left(G(\lambda), \Delta_{u}\right)$ interconnection.

The LFT framework is well suited to handle robust performance questions when the performance specification is small gain in some induced norm. For instance, the $\mathcal{H}_{\infty}$-type robust performance objective specifying that the worst-case $l_{2}$-induced norm from inputs $u$ to outputs $y$ is bounded by 1 , is equivalent to a robust stability question, by adding an additional block $\Delta_{p} y=u$. The proof involves a simple use of Lemma 1.

An extension of the previous concepts to the behavioral case is now presented. In the behavioral setting, the concept of stability is only meaningful if the number of equations is at least equal to the number of variables. In the standard case, such a system is autonomous (see [17]) and stability means that time trajectories decay to zero with time. For the uncertain case we give the following definition.

Definition 2 The GON representation of Figure 4 is well posed if the matrix $D$ is of full coluinn rank. A well posed GON representation has uniform robust stability if there exists $\epsilon>0$, such that given any $\Delta_{S} \in \mathbf{B}_{\Delta_{S}}$ and $l_{2}$ signals $z, w, n$ satisfying the equations with $\|z\|=1$, then $\|n\| \geq \epsilon$.

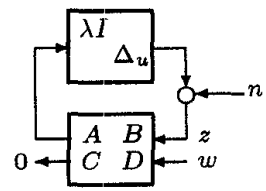

Figure 4: Robust stability analysis in the GON representation

The well posedness condition ensures that there are enough effective constraints so that $z=0$ implies $w=0$. The robust stability definition directly extends Definition 1.

A performance constraint in terms of a $\Delta_{p}$ block can also be incorporated. Furthermore, starting with a regular GON representation, and adding a performance constraint of the type $\Delta_{p} y=u$ between any valid input/output partition automatically gives a square, invertible $D$ matrix and therefore a well posed robust stability question. In this particular case, the robustness analysis question could be resolved by turning the behavioral equations to input/output form. In the next section, however, it will be shown how the behavioral setting allows for additional signal constraints and therefore a richer class of robustness analysis problems. These additional constraints will not, however, affect the full column rank of the $D$ matrix (well posedness condition).

\subsection{Set descriptions for white noise}

We will now show a feature of the uncertain behavioral paradigm that does not have a counterpart in the input output setting: it allows for the inclusion of signal constraints which give approximate descriptions of white noise and therefore permit the analysis of $\mathcal{H}_{2}$ performance in the worst case setting. We begin by considering scalar signals. In [12], sets of signals with a parameterized degree of "whiteness" are defined in terms of constraints of the type

$$
\left|r_{u}(\tau)\right| \leq \gamma r_{u}(0), \quad \tau=1 \ldots T
$$

where $r_{u}(\tau)=\left\langle u, \lambda^{\tau} u\right\rangle$ is the time autocorrelation of a signal $u(t)$. This can be used to give tighter descriptions of empirical disturbances than the usual norm bounded classes. In [12] this is treated in terms of finite records of signals, and conditions on the parameters are given which ensure these sets capture typical behavior of stochastic white noise. Here infinite horizon $l_{2}$ signals will be considered, with autocorrelations performed over all time. Given a linear time invariant stable system $\mathcal{H}(\lambda)=\sum_{t=0}^{\infty} h_{t} \lambda^{t}$ and inputs $u(t),\|u\| \leq 1$ verifying (8), then the norm of the output $y$ verifies

$$
\|y\|^{2} \leq\|\mathcal{H}\|_{2}^{2}+2 \gamma \sum_{\tau=1}^{T}\left|r_{h}(\tau)\right|+2 \sum_{\tau=T+1}^{\infty}\left|r_{h}(\tau)\right|
$$

where $r_{h}(\tau)$ are the autocorrelations of the filter. So for small $\gamma$ (ideally we could use $\gamma=0$ ) and large $T$, the worst case induced norm of the system under the autocorrelation constraints approximates the $\mathcal{H}_{2}$ norm.

These constraints will now be fit into the robust performance problem. The key step is to express signal constraints such as (8) in a fashion compatible with the LFT representation of uncertainty. By virtue of Lemma 1, it seems natural to recast the constraints as norm inequalities. For each $\tau,(8)$ leads to the following pair of constraints $\quad \pm r_{u}(\tau) \leq \gamma r_{u}(0)$ 
Simple manipulations show $\pm r_{u}(\tau)=\|u\|^{2}-\frac{1}{2}\left\|u \mp \lambda^{\tau} u\right\|^{2}$, therefore the constraints of (10) can be written as

$$
2(1-\gamma)\|u\|^{2} \leq\left\|u \pm \lambda^{\tau} u\right\|^{2}
$$

for each $\tau$. Focusing for instance on the plus sign in (11), let $\alpha=$ $\sqrt{2(1-\gamma)}, z=\alpha u, \xi=u+\lambda^{\tau} u$; by Lemma 1 , the constraint is equivalent to the existence of an operator $\delta_{n_{+}},\left\|\delta_{n_{+}}\right\| \leq 1$ such that $\delta_{n_{+}} \xi=z$. To express this by means of an LFT, the variable $u$ must influence both the input and the output of the $\delta_{n_{+}}$operator. The only way to enforce these relations is by writing them in implicit form, as an uncertain behavioral equation as shown in Figure 5.

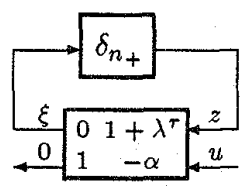

Figure 5: Kernel representation for autocorrelation constraint

A state space representation of the LTI matrix above leads to a GON representation for the constraint. Interconnecting GON representations of an uncertain system, small-gain performance constraints, and the autocorrelation constraints, we obtain a unified GON representation for the constrained robust performance question, which has more equations than manifest variables ("tall" $D$ matrix). This has no counterpart in the standard input/output formulation. In view of the previous discussion of this constrained performance measure, by increasing the number of constraints we approach a robust $\mathcal{H}_{2}$ performance test. This terminology involves a certain abuse of notation, (the uncertain system is not in general LTI), but will be adopted here since it reflects the relevant object from the point of view of applications, i.e. the worst-case gain under white signals. Also mixed $\mathcal{H}_{2} / \mathcal{H}_{\infty}$ performance can be dealt with by constraining only some variables.

The previous construction can be extended to the multivariable case, by considering the autocorrelation matrix of a (column) vector valued signal $u \in l_{2}^{m}, R_{u}(\tau)=\sum_{t=-\infty}^{\infty} u(t+\tau) u(t)^{*}$. For $u$ to be white, $R_{u}(\tau)$ must be 0 for $\tau \neq 0$, and $R_{u}(0)$ must be a multiple of the identity matrix. These matrix constraints can be reduced to a number of scalar constraints, and treated as before.

For the case $\tau \neq 0$, a simpler method can be obtained by using Lemma 2. The key observation is that

$$
R_{u}(\tau)=0 \Longleftrightarrow \forall \eta \in \mathbb{C}^{m},\left\langle\eta^{*} u, \eta^{*} \lambda^{\tau} u\right\rangle=0
$$

A similar procedure as in the scalar case leads to the equivalent constraints

$$
2\left\|\eta^{*} u\right\|^{2} \leq\left\|\eta^{*}\left(u \pm \lambda^{\tau} u\right)\right\|^{2} \quad \forall n \in \mathbb{C}^{m}
$$

By Lemma 2, each constraint (12) is equivalent to the existence of an operator $\delta_{n} I,\left\|\delta_{n} I\right\| \leq 1$, such that $\delta_{n} I \xi=z$, where $\xi=u \pm \lambda^{\tau} u$, $z=\sqrt{2} u$. This is entirely analogous to the scalar case and can be readily expressed as an uncertain behavior involving the $\delta_{n} I$ blocks.

\subsection{Integral quadratic constraints}

Both the small-gain performance constraints and the autocorrelation constraints described above are special cases of the integral quadratic constraints (IQCs) of Megretski ([8] and [S]). The general form of these constraints, in the frequency domain, is

$$
\int_{-\pi}^{\pi} \hat{w}^{*}\left(e^{j \theta}\right) G\left(e^{j \theta}\right) \hat{w}\left(e^{j \theta}\right) d \theta \leq 0
$$

where $\hat{w}$ is the Fourier transform of a vector signal $w$, and $G=G^{*}$ is a weighting function.

We will now show that these general IQCs can be written (for finite dimensional weighting functions) in a GON representation. By decomposing the hermitian matrix $G$ into positive and negative parts as $G=P^{*} P-Q^{*} Q$, condition (13) is equivalent to

$$
\left\|P\left(e^{j \theta}\right) w\left(e^{j \theta}\right)\right\|_{2}^{2} \leq\left\|Q\left(e^{j \theta}\right) w\left(e^{j \theta}\right)\right\|_{2}^{2}
$$

Defining $z=P w, \xi=Q w$, by Lemma 1 the constraint (14) is equivalent to the existence of $\Delta_{I Q C},\left\|\Delta_{I Q C}\right\| \leq 1$ such that $z=$ $\Delta_{I Q C} \xi$. The result is depicted in Figure 6.

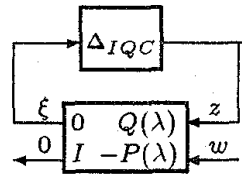

Figure 6: IQC as an uncertain behavioral equation

Assuming that the weight functions $P, Q$ can be realized by finite dimensional filters, writing state space realizations for $P, Q$ a GON representation with constant matrix $M$ can be obtained.

To summarize, the uncertain behavioral paradigm seems to be the natural setting where various descriptions of uncertainty can coexist. It allows for parametric and dynamic uncertainty, as well as signal constraints designed to characterize disturbances or to specify performance requirements. The robustness analysis for these situations can then be reduced to a robust stability question.

\section{Convex Tests for Robustness}

In this section, conditions for robustness analysis in uncertain behavioral setup will be provided. The starting point is the GON representation of Figure 4, which will be assumed to be well posed, (i.e $D$ is a full column rank $p \times q$ matrix). Two behavior preserving transformations reduce the GON representation to a simpler form.

The first is to pre-multiply the equations by an invertible matrix $P$ chosen so that $P D=\left[\begin{array}{c}I_{q} \\ 0\end{array}\right]$. Let $P C=\left[\begin{array}{c}\hat{C}_{1} \\ \hat{C}_{2}\end{array}\right]$. The second step is to perform an output injection using the first $q$ equations to set $B=0$. The result is depicted in Figure 7 , where $\hat{A}=A-B \hat{C}_{1}$.

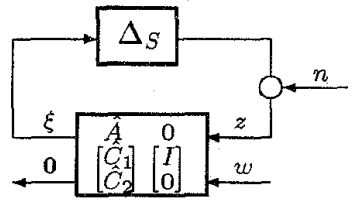

Figure 7: Final version of GON representation

In this new representation, returning to Definition 4, it is clear that the variable $w$ has been decoupled from the problem: if signals $z, n$ and a perturbation $\Delta_{S}$ can be found to satisfy the equations $\left(I-\Delta_{S} \hat{A}\right) z=n, \hat{C}_{2} z=0$, then $w$ is automatically determined from $z$. We summarize the previous steps in the following statement:

Proposition 1 Given a well posed GON representation $\left(\Delta_{S}, M\right)$, where $M=\left[\begin{array}{ll}A & B \\ C & D\end{array}\right]$, if $P$ is such that $P D=\left[\begin{array}{c}I_{q} \\ 0\end{array}\right] \quad P C=\left[\begin{array}{c}\hat{C}_{1} \\ \hat{C}_{2}\end{array}\right]$ and $\hat{A}=A-\hat{C}_{1} B$, then the GON system is uniformly robustly stable if and only if there exists $\epsilon>0$ such that if $\hat{C}_{2} z=0,\|z\|=1$, $\Delta_{S} \in \mathbf{B}_{\Delta_{\mathbf{S}}}$, then $\left\|\left(I-\Delta_{S} \hat{A}\right) z\right\| \geq \epsilon$.

The resulting condition makes the problem look like a usual ( $\mu$ type) structured uncertainty analysis question on the matrix $\hat{A}$, except for the additional constraint $\hat{C}_{2} z=0$. In what follows, we will show how appropriate extensions of the $\mu$-analysis machinery can be given to provide robustness conditions in this case. In particular, we will focus on extending the $\mu$ upper-bound condition in terms of an LMI. The condition will always be sufficient for uniform robust stability, we will later return to the question of necessity.

For a general delta structrure $\Delta$ of the form described in (2) we will consider positive scaling matrices $X$ that commute with the elements in $\boldsymbol{\Delta}$, of the form

$X=\operatorname{diag}\left[X_{1}, \ldots, X_{L}, x_{L+1} I_{m_{1}}, \ldots, x_{L+F} I_{m_{F}}\right], X=X^{*}>0$,

$\mathbf{X}$ will denote the set of such matrices $\left(\mathbf{X}_{\mathrm{B}}\right.$ or $\mathbf{X}_{\mathbf{u}}$ when specializing on $\Delta_{S}$ or $\Delta_{u}$ ). For convenience, from now on we will assume the system is already in the simplified form of Figure 7, and write $A, C$ instead of $\hat{A}, \hat{C}_{2}$. Without loss of generality we can also assume that $\mathrm{C}$ is of full row rank.

The following is the sufficiency result, which holds for an arbitrary $\Delta$ structure. 
Theorem 1 Let $A, C$ be given, define $C_{\perp}$ as a matrix with rows spanning the orthogonal complement of the rows of $C$. If there exists $X \in \mathbf{X}$ such that

$$
C_{\perp}\left(A^{*} X A-X\right) C_{\perp}^{*}<0
$$

then there exists $\epsilon>0$ such that if $\|z\|=1, C z=0, \Delta \in \mathbf{B}_{\Delta}$, then $\|(I-\Delta A) z\| \geq \epsilon$.

Proof: Fix $X>0$ which solves (15). By continuity, we can find $\alpha<1$ such that $C_{\perp}\left(A^{*} X A-\alpha X\right) C_{\perp}^{*}<0$. Choose $z, n$ such that

$$
\begin{aligned}
(I-\Delta A) z & =n \quad\|z\|=1 \\
C z & =0
\end{aligned}
$$

By (17), $z=C_{\perp}^{*} u$; defining $\bar{z}=X^{\frac{1}{2}} z, \bar{n}=X^{\frac{1}{2}} n$, then (16) gives $\left(I-\Delta X^{\frac{1}{2}} A X^{-\frac{1}{2}}\right) \bar{z}=\bar{n}$. Now

$\begin{aligned}\left\|\Delta X^{\frac{1}{2}} A X^{-\frac{1}{2}} \bar{z}\right\|^{2} & \leq\left\|X^{\frac{1}{2}} A C_{\perp}^{*} u\right\|^{2}= \\ \sum_{t=-\infty}^{\infty} u(t)^{*} C_{\perp} A^{*} X A C_{\perp}^{*} u(t) & \leq \alpha \sum_{t=-\infty}^{\infty} u(t)^{*} C_{\perp} X C_{\perp}^{*} u(t)=\alpha\|\bar{z}\|^{2}\end{aligned}$

Therefore $\|\bar{n}\| \geq\|\bar{z}\|-\left\|\Delta X^{\frac{1}{2}} A X^{-\frac{1}{2}} \bar{z}\right\| \geq(1-\sqrt{\alpha})\|\bar{z}\|$. Since $\|\bar{z}\| \geq \underline{g}\left(X^{\frac{1}{2}}\right)\|z\|,\|z\|=1$, and $\|\bar{n}\| \leq \bar{\sigma}\left(X^{\frac{1}{2}}\right)\|n\|$, then

$$
\|n\| \geq(1-\sqrt{\alpha}) g\left(X^{\frac{1}{2}}\right) / \bar{\sigma}\left(X^{\frac{1}{2}}\right)
$$

The uniform robust stability is therefore satisfied by choosing $\epsilon$ as the right hand side of (18), which is independent of $\Delta$.

The previous result gives us a tractable way of assessing robustness of an uncertain behavioral system in terms of a convex problem (i.e. solution of a linear matrix inequality). To consider the potential conservatism of this condition, we now turn to the question of necessity. Recent results by Shamma [14] and Megretski ([8], [9]) have shown that in the case of full blocks in the uncertainty structure, sufficient conditions in terms of $X$-scalings on the uncertainty are in fact necessary for robust stability when the blocks are allowed to be arbitrary linear operators in $l_{2}$ space. We can state a similar result for the uncertain behavioral setup.

Theorem 2 In the conditions of Theorem 1 , with $\Delta$ varying in the set of arbitrary structured linear operators in $l_{2}^{n}$, if the GON representation has uniform robust stability, then $\exists X \in \mathbf{X}$ such that $C_{\perp}\left(A^{*} X A-X\right) C_{\perp}^{*}<0$

In view of the duality between IQCs and uncertain behavioral descriptions that was shown in section 3 , the proof of this result is essentially a reformulation of the S-procedure losslessness results of Megretski and Treil [10]. The main extension that is needed is to capture the $\delta I$ blocks, which are not described by scalar valued IQCs. In this constant matrix case, the result is closely related to earlier work in [1]. The full proof is deferred to a future paper [13].

It is interesting to note a connection between condition (15) and previous work on stabilization of input-output LFT systems in [7], where it is shown that there exists a solution to (15) if and only if there exists an output injection matrix $L$ that makes the matrix $A+$ $L C Q$-stable. This property is referred to as $Q$-detectability. From this point of view, our robust stability condition, which implies $\operatorname{Ker}\left[\begin{array}{c}I_{n}-\Delta A \\ C\end{array}\right]=0$, can be interpreted as an extension of the PBH test for $Q$-detectability.

The previous necessity result is not entirely satisfactory because it requires every block in the $\Delta$ structure to be allowed to vary in the class of arbitrary linear operators. This includes the first $\delta I$ block, which in a GON representation is usually specified to be $\lambda I$. To analyze this issue, assume that $\Delta_{S}=\operatorname{diag}\left[\lambda I \Delta_{u}\right]$, where $\Delta_{u}$ varies in the class of structured, otherwise arbitrary linear operators. The matrices $A, C$ and the signal $z$ can be partitioned in accordance with $\Delta_{S}$

$$
A=\left[\begin{array}{cc}
A_{n} & A_{n u} \\
A_{u n} & A_{u}
\end{array}\right], C=\left[\begin{array}{ll}
C_{n} & C_{u}
\end{array}\right]
$$

We will now make the assumption that $C_{\mathfrak{n}}=0$, and show that (15) is still necessary under the restricted class of perturbations. This assumption is not overly restrictive: in the next section it is shown this is satisfied when considering a robust $\mathcal{H}_{2}$ performance problem. The general case is, however, still of interest and will be analyzed in further detail in [13]. We have the following result:
Theorem 3 Let $A, C$ be as in (19), $C_{n}=0, G(\lambda) \triangleq A_{u}+$ $A_{u n} \lambda\left(I-\lambda A_{n}\right)^{-1} A_{n u}$. Then the following are equivalent:

(i) $\exists \epsilon>0$ such that if $\Delta_{S} \in \mathbf{B}_{\Delta_{S}},\|z\|=1, C z=0$, then $\left\|\left(I-\Delta_{S} A\right) z\right\| \geq \epsilon$

(ii) $\rho\left(A_{n}\right)<1$, and $\exists \epsilon>0$ such that if $\Delta_{u} \in \mathbf{B}_{\Delta_{u}}$, $\left\|z_{u}\right\|=1, C_{u} z_{u}=0$, then $\left\|\left(I-\Delta_{u} G(\lambda)\right) z_{u}\right\| \geq \epsilon$

(iii) $\exists X_{u} \in \mathbf{X}_{\mathbf{u}}$, such that $C_{u \perp}\left(G^{*} X_{u} G-X_{u}\right) C_{u \perp}^{*}<0$ (20)

(iv) $\exists X_{S} \in \mathbf{X}_{\mathbf{S}}$, such that $C_{\perp}\left(A^{*} X_{S} A-X_{S}\right) C_{\perp}^{*}<0$

Proof: $(i) \Rightarrow\left(\right.$ ii). First, if $\rho\left(A_{n}\right) \geq 1$, let $a_{0}, z_{0}$ be the eigenvalue, unit eigenvector achieving the spectral radius, and choose $\Delta_{S}=$ $\operatorname{diag}\left[a_{0}^{-1} \lambda I, 0\right], z_{u}=0, z_{n}(t)=\frac{1}{\sqrt{t_{0}}} z_{0}, 0 \leq t<t_{0}, 0$ otherwise. This gives $\|z\|=1$, and $\left\|\left(I-\Delta_{u} A\right) z\right\|^{2}=\frac{2}{t_{0}}$ which can be made arbitrarily small, contradicting $(i)$. So $\rho(A)<1$. Given $z_{u},\left\|z_{u}\right\|=$ $1, C_{u} z_{u}=0$, and $\Delta_{u} \in \mathbf{B}_{\Delta_{u}}$, feeding $z_{u}$ to $G(\lambda)$ gives by the stability of $A_{n}$, an $l_{2}$ state signal $z_{n}$. Choosing $\Delta_{S}=\operatorname{diag}\left[\lambda I, \Delta_{u}\right]$, $z=\left[z_{n}^{\prime}, z_{u}^{\prime}\right]^{\prime}$ we find $C z=0$ therefore

$$
\left\|\left(I-\Delta_{u} G(\lambda)\right) z_{u}\right\|=\left\|\left(I-\Delta_{S} A\right) z\right\| \geq \epsilon
$$

(ii) $\Rightarrow$ (iii) The constraint (20) is of the form $\Phi<0$ where $\Phi$ is an operator on $l_{2}$, this meaning that the quadratic form $\langle\Phi u, u\rangle$ is negative definite on $l_{2}$. The proof of this result is essentially the same as Theorem 2 , and is deferred to [13].

(iii) $\Rightarrow$ (iv) By pre and post multiplication of (20) by an invertible matrix we can assume without loss of generality that
$C_{u \perp} X_{u} C_{u \perp}^{*}=I$. Then $(20)$ can be rewritten as $\|F(\lambda)\|_{\infty}<1$, with $F(\lambda)=X_{u}^{\frac{1}{2}} G(\lambda) C_{u \perp}^{*}$, which has state space realization

$$
\left[\begin{array}{ll}
A_{F} & B_{F} \\
C_{F} & D_{F}
\end{array}\right]=\left[\begin{array}{cc}
A_{n} & A_{n u} C_{u \perp}^{*} \\
X_{u}^{\frac{1}{2}} A_{u n} & X_{u}^{\frac{1}{2}} A_{u} C_{u \perp}^{*}
\end{array}\right]
$$

It is well known that the test $\|F(\lambda)\|_{\infty}<1$ is equivalent to the existence of a solution $X_{1}>0$ to the LMI

$$
\left[\begin{array}{cc}
A_{F} & B_{F} \\
C_{F} & D_{F}
\end{array}\right]^{*}\left[\begin{array}{cc}
X_{1} & 0 \\
0 & I
\end{array}\right]\left[\begin{array}{cc}
A_{F} & B_{F} \\
C_{F} & D_{F}
\end{array}\right]-\left[\begin{array}{cc}
X_{1} & 0 \\
0 & I
\end{array}\right]<0
$$

Substituting the expressions for $A_{F}, B_{F}, C_{F}, D_{F}$, and using the fact $C_{u \perp} X_{u} C_{u \perp}^{*}=I,(21)$ leads to

$$
\left[\begin{array}{cc}
I & 0 \\
0 & C_{u \perp}
\end{array}\right]^{*}\left(A^{*}\left[\begin{array}{cc}
X_{1} & 0 \\
0 & X_{u}
\end{array}\right] A-\left[\begin{array}{cc}
X_{1} & 0 \\
0 & X_{u}
\end{array}\right]\right)\left[\begin{array}{cc}
I & 0 \\
0 & C_{u \perp}^{*}
\end{array}\right]<0
$$

We observe that since $C=\left[\begin{array}{ll}0 & C_{u}\end{array}\right], C_{\perp}=\left[\begin{array}{cc}I & 0 \\ 0 & C_{u \perp}\end{array}\right]$, so setting $X_{S}=\operatorname{diag}\left[X_{1} X_{u}\right]$ proves $(i v)$.

(iv) $\Rightarrow(i)$ : This is a special case of Theorem 1 .

\section{Application to Robust $\mathcal{H}_{2}$ Analysis}

In this section, the machinery developed so far will be focused specifically on the question of obtaining robust performance guarantees in the presence of white noise disturbances. As discussed earlier, this problem can be approximated by a finite number of autocorrelation constraints, which can be recast in our framework. The problem, depicted in Figure 8, is to test whether the worstcase $l_{2}$ gain from $d$ to $e$ in the presence of uncertainty $\Delta_{u}$ is less than $\beta$, when the input signal $d$ is forced to satisfy "whiteness" constraints of the form discussed in section 3,2. These constraints are represented on the left in the picture; referring back to 3.2 , the matrix can $P$ can be chosen to be constant.

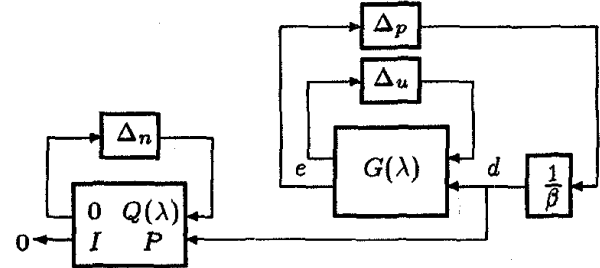

Figure 8: Robust $\mathcal{H}_{2}$ Analysis

Writing state-space realizations

$$
G(\lambda)=\left[\begin{array}{c|cc}
A_{G} & B_{G 1} & B_{G 2} \\
\hline C_{G 1} & D_{G 11} & D_{G 12} \\
C_{G 2} & D_{G 21} & D_{G 22}
\end{array}\right] \quad Q(\lambda)=\left[\begin{array}{c|c}
A_{Q} & B_{Q} \\
\hline C_{Q} & D_{Q}
\end{array}\right]
$$


the whole system can be reduced to a GON representation of the form of Figure 4. Choosing $w=d$, and $\Delta_{S}=$ $\operatorname{diag}\left[\lambda I, \lambda I, \Delta_{u}, \Delta_{p}, \Delta_{n}\right]$ gives after some algebra the matrices

$$
\left[\begin{array}{c|c}
A & B \\
\hline C & D
\end{array}\right]=\left[\begin{array}{ccccc|c}
A_{G} & 0 & B_{G 1} & 0 & 0 & B_{G 2} \\
0 & A_{Q} & 0 & 0 & 0 & B_{Q} \\
C_{G 1} & 0 & D_{G 11} & 0 & 0 & D_{G 12} \\
C_{G 2} & 0 & D_{G 12} & 0 & 0 & D_{G 22} \\
0 & C_{Q} & 0 & 0 & 0 & D_{Q} \\
\hline 0 & 0 & 0 & I & 0 & \beta I \\
0 & 0 & 0 & 0 & I & P
\end{array}\right]
$$

Here $D$ is a full column rank matrix, and by the form of $C$ it is clear that after the transformations described in section 4 , the matrix $\hat{C}_{2}$ is of the form [0 $\left.C_{u}\right]$, as in the assumption of theorem 3. This implies that, provided the uncertainty $\Delta_{u}$ is allowed to vary in the class of arbitrary linear operators $\left(\Delta_{p}, \Delta_{n}\right.$ already have this property), LMI (15) provides a non-conservative, convex test for robust performance under the autocorrelation constraints. Varying the parameter $\beta$ and testing for feasibility in the LMI, we can approximate the infimum $\beta_{\text {opt }}$ of the values for which (15) is satisfied; this is a measure of the worst-case gain under uncertainty $\Delta_{u}$ and autocorrelation constraints. Asymptotically, as the number of constraints increases, the process converges down to a robust $\mathcal{H}_{2}$ performance measure, so that a finite number of constraints always gives an upper bound.

We will present two very simple examples to demonstrate the machinery, applied to problems involving the $\mathcal{H}_{2}$ norm.

The first example, with no uncertainty, consists of calculating the $\mathcal{H}_{2}$ norm of the transfer function $H(\lambda)=\frac{1}{\lambda-2}$ using this approach. There are of course exact ways to compute the $\mathcal{H}_{2}$ norm, which give a result of 0.577 ; this example is included for verification purposes.

The process described above was performed with a number $T$ of autocorrelation constraints (for $\gamma=0$ ). The feasibility of LMI (15) was checked using the software package LMI-lab. Figure 9 depicts $\beta_{\text {opt }}$ as a function of $T$. Starting at $T=0$ with the unconstrained $\left(\mathcal{H}_{\infty}\right)$ norm which is $1, \beta_{\text {opt }}$ asymptotically converges to the $\mathcal{H}_{2}$ norm, as expected, at a rate consistent with the bound (9).

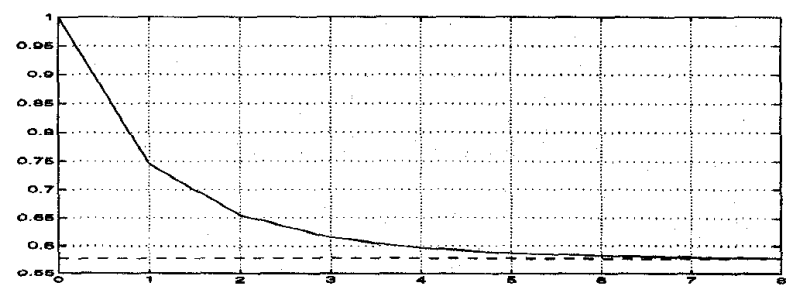

Figure 9: Approximation to $\mathcal{H}_{2}$ norm

The next example incorporates uncertainty into the system description. Let $H(\lambda)=\frac{1}{\lambda-2}+\Delta_{u}$, where $\Delta_{u}$ is assumed to be an LTI perturbation whose $\mathcal{H}_{\infty}$ norm is bounded by 1 . The worst case $\mathcal{H}_{2}$ norm of $H(\lambda)$ can be obtained in the frequency domain:

$$
\sup _{\left\|\Delta_{u}\right\|_{\infty} \leq 1}\|H\|_{2}=\left(\frac{1}{2 \pi} \int_{-\pi}^{\pi}\left(\left|\frac{1}{e^{j \theta}-2}\right|+1\right)^{2} d \theta\right)^{\frac{1}{2}}=1.554
$$

The procedure described above can be performed to calculate worst case induced norme under correlation constraints. Figure (10) shows the corresponding plot of $\beta_{\text {opt }}$ as a function of $T$.

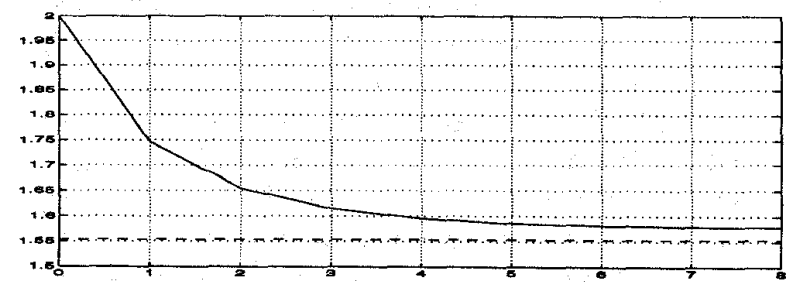

Figure 10: Induced norm of uncertain system

There appears to be a gap (of about 1 or $2 \%$ ) between the asymptotic gain and the value calculated in (23). This can be explained by the fact that the analytical solution is based on $\Delta_{u}$ being an
LTI perturbation, while the LMI approach is exact only for the larger class of arbitrary linear time-varying operators.

\section{Conclusions}

The uncertain behavioral representation has been shown to be an attractive general setup for robust control, where various forms of system uncertainty, performance requirements and signal constraints can be expressed. Specifically, general robust performance problems under parametric and dynamic uncertainty in the plant and integral quadratic constraints are recast as robust stability problems of a GON representation. This includes the particular case of autocorrelation constraints for $\mathcal{H}_{2}$ performance.

Convex tests (15) for robust stability of the GON representations in terms of LMIs were obtained, which are exact for the case where the $\Delta$ perturbations are arbitrary operators. In this respect, these results are equivalent to those obtained dealing directly with IQCs ([8], [9]), with an extension to account for $\delta I$ blocks.

The LFT framework is advantageous, however, to express more highly structured uncertainty not captured by IQCs, such as real parametric uncertainty. In these cases condition (15) is conservative, as is well known for the standard $\mu$-analysis case, and exact conditions will have an increased computational complexity.

The ultimate impact of this framework, however, should be much broader and allow for a more unified approach to modeling, analysis, model validation, system identification, and perhaps even controller synthesis and system design.

\section{Acknowledgements}

The authors would like to acknowledge A. Rantzer and A. Megretsky for numerous helpful discussions, and Yun Huang for helping in the computational aspects of this paper. We would like to acknowledge AFOSR, NSERC, and NSF as funders of this work.

\section{References}

[1] Bercovici H., Foias C., Tannenbaum A., Structured Interpolation Theory, Operator Theory Adv, and App., 47, pp:195-220, 1990.

[2] Bernstein D.S Haddad W.H., LQG Control with an $\mathcal{H}_{\infty}$ Performance Bound: A Ricatti Equation Approach, IEEE Trans. Automatic Control, Vol 34, 3,pp. 293-305, 1989.

[3] D'Andrea R., Paganini F., Doyle J.C., Uncertain Behavior, Proceedings 1993 CDC, San Antonio, Texas, pp. 3891-3896.

[4] D'Andrea. R., Paganini F., Interconnection of Uncertain Behavioral Systems for Robust Control, Proceedings 1993 CDC, San Antonio, Texas, pp. 3642-3647.

[5] Hall S., How J., Mixed $\mathcal{H}_{2} / \mu$ Performance Bounds using Dissipation Theory, Proc. 1993 CDC, San Antonio, TX, pp 1536-1541.

[6] Khargonekar P., Rotea $M$., Mixed $\mathcal{H}_{2} / \mathcal{H}_{\infty}$ Control: A convex Optimization Approach, IEEE Trans. A.C., Vol 36, 7,pp. 824$837,1991$.

[7] Lu W.M., Zhou K., Doyle J.C., Stabilization of LFT Systems, Proceedings 1991 CDC, Brighton, England, pp. 1239-1244.

[8] Megretski A., Necessary and Sufficient Conditions of Stability: A Multiloop Generalization of the Circle Criterion, IEEE Trans. Automatic Control, Vol 38, 5, 1993.

[9] Megretski A., S-Procedure in Optimal Non-Stochastic Filtering, Tech. Rep. Code Trita/Mat-92-0015, Royal Inst. of Tech., Sweden.

[10] Megretski A., and Treil, S., S-procedure and power distribution inequalities: $A$ new method in optimization and robustness of uncertain systems, Mittag-Leffler Inst., Rep. No 1, 1990/91.

[11] Packard A., Doyle J.C., The Complex Structured Singular Value, Automatica, Vol. 29, No. 1, pp. 71-109, 1993.

[12] Paganini F., Set Descriptions of White Noise and Worst Case Induced Norms, Proceedings 1993 CDC, San Antonio, Texas, pp. 3658-3663.

[13] Paganini F., Doyle J.C., in preparation.

[14] Shamma, J.,Robust Stability with Time Varying Structured Un. certainty, to appear IEEE Trans. A.C.

[15] Stoorvogel A.A., The Robust $\mathcal{H}_{2}$ Control Problem: A WorstCase Design, IEEE Trans. A.C., Vol 38, 9,pp. 1358-1370, 1993.

[16] Sznaier M., An (Almost) Exact Solution to General SISO Mixed $\mathcal{H}_{2} / \mathcal{H}_{\infty}$ Problems via Convex Optimization, Proceedings 1993 ACC, San Francisco, CA, pp. 250-254.

[17] Willems J.C., Paradigms and Puzzles in the Theory of Dynamical Systems, IEEE Trans. A.C., Vol. 36, pp. 259-294, 1991

[18] Zhou K., Doyle J., Glover K., Bodenheimer,B., Mixed $\mathcal{H}_{2}$ and $\mathcal{H}_{\infty}$ Control, Proc. 1990 ACC, San Diego, CA, pp 2502-2507. 